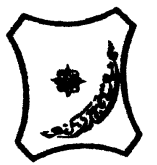

Bayero Journal of Pure and Applied Sciences, 8(2); 135 - 137

Received: November, 2015

Accepted: November, 2015

ISSN: 2006 - 6996

\title{
UNUSUAL OCCURRENCE OF CONGENITAL HYPOTHYROIDISM IN A SET OF SAME SEX TRIPLETS: CHALLENGES OF DIAGNOSIS IN A RESOURCE POOR SETTING
}

\author{
1,2 Abdulkadir, M.B., ${ }^{1,2}$ Obasa, T.O., ${ }^{2}$ Ogunkanbi, S.O., ${ }^{1,2}$ Adeboye, M.A.N., ${ }^{1,2}$ Adesiyun, \\ 0.0., 1,2Mokuolu, 0.A.
}

1. Department of Paediatrics and Child Health, University of Ilorin, Ilorin, Kwara State, Nigeria

2. Department of Paediatrics and Child Health, University of Ilorin Teaching Hospital, Ilorin, Kwara State, Nigeria

Email address: docmohng@gmail.com,+2348065754333

\begin{abstract}
:
Congenital hypothyroidism is the most common endocrinological disorder in children. Genetic and intrauterine factors have been implicated in its aetiology. The aim of this study was to describe an unusual occurrence of congenital hypothyroidism in a set of same sex triplets and challenges associated with the diagnosis. This is a review of a set of female triplets delivered at 34 weeks gestational age and noticed to have reduced activity, hypotonia and poor suck. On testing for thyroid hormones the patients were found to be hypothyroid. However this diagnosis was delayed until the $\sigma^{\text {th }}$ week of life as a result of difficulties in running the test. The subjects of the study were placed on thyroxine supplementation and have been doing well. Congenital hypothyroidism can occur in multiple order pregnancies and clinicians should have a high index of suspicion for its occurrence.

Keywords: Congenital hypothyroidism; Triplets; Diagnosis
\end{abstract}

\section{INTRODUCTION}

Hypothyroidism is one of the most common causes of preventable mental retardation globally (Delange, 1997). Early diagnosis is critical in preventing the devastating consequences of the disease (Delange, 1997). In developed countries, newborn screening programmes have been proven to be an efficient and effective tool in its early diagnosis (Delange, 1997; Mikelsaar and Viikmaa, 1999). Neonatal screening programmes have reported a prevalence of 1 in 4000 infants, with the prevalence being lower in BlackAmericans (Fisher, 1983). However, in most developing countries, newborn screening programmes are yet to be developed. Thus, diagnosis requires a high index of suspicion using suggestive clinical features. The most common cause $(85 \%)$ of congenital hypothyroidism is thyroid dysgenesis with a majority $(98 \%)$ of these cases occurring sporadically (Bernstein, 2011). About $2 \%$ of the cases are familial (Park and Chatterjee, 2005). Candidate genes for the familial form include genes for thyroid stimulating hormone (TSH) receptor, thyroid peroxidase, thyroglobulin, the sodium iodide symporter, pendrin, and most recently, thyroid oxidase 2 (Park and Chatterjee, 2005). Occurrence of similar conditions among triplets raises a strong suspicion of genetic / environmental contributions to the disorder, rather than the sporadic form which is commoner globally. We present an unusual occurrence of congenital hypothyroidism in a set of female triplets.

\section{MATERIALS AND METHODS}

This is a retrospective case review of congenital hypothyroidism in a set of triplets seen in 2013 at the Neonatal Intensive Care Unit (NICU) of the University of Ilorin Teaching Hospital (UITH), a tertiary hospital in north-central Nigeria. Case folders were retrieved from the health records department. Relevant information was extracted, which included age, gender, history and examination findings at presentation, investigations and treatment given and details of subsequent follow up.

\section{RESULTS}

Babies OA I, II, and III were 34 week gestational age (GA) all female triplets referred to the NICU from a secondary health facility at 30 minutes of age on account of low apgar scores.

They were delivered to a 38-year old now para 3+0 (4 alive) woman via spontaneous vertex delivery. 
The pregnancy was planned and booked at a peripheral hospital at 20-weeks' gestation. She had not received any fertility medications prior to pregnancy. The mother had not been diagnosed with any chronic illness (Hypertension, diabetes or hyperthyroidism) and was not on any medications asides haematinics and intermittent preventive therapy for malaria with sulphadoxine-Pyrimethamine. She had 4 antenatal visits which had been essentially uneventful. Mother had presented in labour at the booking hospital, with a 24-hour history of liquor drainage and a 6 hour history of progressive labour pains.

The $1^{\text {st }}$ of the triplets was delivered shortly after presentation at the referring hospital and required only airway clearance with documented apgar scores of 5(One minute) and 8(Five minutes). The second triplet was delivered about 10 minutes later and had no respiratory effort requiring airway clearance and bag and mask ventilation for about 2 minutes. Apgar scores for the $2^{\text {nd }}$ triplet were $3^{1}$ and $5^{5}$ with an extended Apgar score of $7^{10}$. The third triplet was delivered about 10 minutes after the first with Apgar scores of $5^{1}$ and $7^{5}$. They were kept warm and transferred to this hospital.

The mothers' first pregnancy was delivered of a male child at term with no complications. The child is currently 6 years old and doing well. There was a history of early neonatal death for her second pregnancy about 3 years ago in which the child died within 36 hours of delivery following a failure to cry at birth with weak effort at the secondary health facility. Mother is a teacher with Ordinary National Diploma (OND) while father is a police officer with same qualification.

On examination at admission all three neonates were profoundly lethargic with generalized hypotonia and poor cortical responses. They had a weak cry and depressed primitive reflexes (rooting, sucking, Moro). They were in respiratory distress and had tachycardia. An initial diagnosis of severe perinatal asphyxia with a risk for sepsis was made and they were commenced on parenteral antibiotics (cefuroxime and gentamycin) and the necessary supportive care (intravenous fluids, oxygen, Nil per oral , monitoring of urine output and vital signs).

Respiratory distress resolved within 24hrs of admission though babies remained floppy and by the third day they were commenced on feeds via orogastric tube. However throughout this period, they were noted to still be profoundly lethargic and markedly hypotonic. There was no jaundice, abdominal distension and they had passed meconium within 24 hours of delivery.

By the $6^{\text {th }}$ day of life; they were considered well enough to commence cup and spoon feeds however these were not tolerated as a result of a weak suck and swallow reflexes. Subsequent attempts at feeding via that route resulted in aspiration of feeds. They were subsequently maintained on feeds via orogastric tube and over the next 2 weeks they gained weight at a rate of triplet $1(15 \mathrm{~g} / \mathrm{kg}$ per day) triplet 2 $(12 \mathrm{~g} / \mathrm{kg} /$ day $)$ and triplet $3(18 \mathrm{~g} / \mathrm{kg} /$ day $)$.

On account of persistent floppiness, sluggishness, weak cry and poor oral feeding in spite of consistent weight gain, a transfontanelle ultrasound and thyroid function test were requested. Transfontanelle ultrasound was done on the $15^{\text {th }}$ day of life and revealed bilateral grade 2 intraventricular haemorrhage in all three. However thyroid function tests could not be done until 2 weeks later as a result of a laboratory policy of pooling samples before running the tests.

By the $6^{\text {th }}$ week of life the triplet had gained weight remarkably. Thyroid function test results were received as shown in Table 1.

Table 1: Thyroid function test results of the patients before commencement of thyroxine

\section{$\begin{array}{llll}\text { Reference ranges } & \text { Triplet } 1 & \text { Triplet } 2 & \text { Triplet } 3\end{array}$}

\begin{tabular}{lcccc}
\hline Free serum $\mathrm{T}_{3}(\mathrm{pmol} / \mathrm{L})$ & $3.7-8.6$ & 2.8 & 2.9 & 2.2 \\
Free serum $\mathrm{T}_{4}(\mathrm{pmol} / \mathrm{L})$ & $12-33$ & 11.8 & 10.1 & 10.3 \\
Serum TSH ( $\mathrm{mIU} / \mathrm{L})$ & $1.7-9.1$ & 2.55 & 2.6 & 1.83 \\
\hline
\end{tabular}

Table 2: Thyroid function test results four weeks after commencement of thyroxine

$\begin{array}{llll}\text { Reference ranges } & \text { Triplet } 1 & \text { Triplet } 2 & \text { Triplet } 3\end{array}$

\begin{tabular}{|c|c|c|c|c|}
\hline Free serum $T_{3}(\mathrm{pmol} / \mathrm{L})$ & $3.7-8.6$ & 3.9 & 4.5 & 4.2 \\
\hline Free serum $\mathrm{T}_{4}(\mathrm{pmol} / \mathrm{L})$ & $12-33$ & 14.2 & 15.6 & 15.5 \\
\hline Serum TSH ( mIU/L) & $1.7-9.1$ & 2.8 & 2.7 & 3.4 \\
\hline
\end{tabular}


L-Thyroxine was commenced at $15 \mu \mathrm{g} / \mathrm{kg}$ daily for all triplets. One week later, activity had improved and they were now tolerating cup and spoon feeds with minimal improvement in the tone. Over the next 2 weeks, all triplets were successfully discharged on Lthyroxine and have been tolerating oral feeds. A repeat assay of thyroid hormones four weeks after commencement of L-thyroxine revealed normal values of the thyroid hormones (Table 2). Activity and tone were subsequently noticed to have improved and the children are currently being followed up at the endocrinology clinic where they are currently doing fine.

\section{DISCUSSION}

Despite thyroid dysgenesis being the commonest cause of congenital hypothyroidism globally, most cases are sporadic with only $10-15 \%$ being inherited, either as autosomal recessive or autosomal dominant (Fisher, 1983). There have been several reports of congenital hypothyroidism in twins however there are few reports in triplets and higher order pregnancies (Olivieri et al., 2007). To the best of the knowledge of the authors,

\section{REFERENCES}

Kliegman, R. M., Stanton, B.F., St-Geme, J., Schor, N., and Behrman, R.E.(2011). Nelson Textbook of Pediatrics.18 th edition. Philadelphia: Elsevier/Saunders, Pp 1894-1915.

Delange, F. (1997). Neonatal Screening for Congenital Hypothyroidism Results and Perspectives. Hormone Research in Paediatrics, 48(2): 5161.

Fisher, D. A.(1983). Second International Conference on NeonatalThyroid Screening: Progress report. The Journal of Pediatrics, 102(5): 653654.

Mikelsaar, R. V. and Viikmaa, M. (1999). Neonatal Thyroid-Stimulating Hormone Screening as an this is the first report of congenital hypothyroidism in a set of triplets in Africa. Oliviera et al (2007) in a report of a 12 year screening programme in Italy reported only one case of congenital hypothyroidism in a set of triplets (two females and one male). While detailed genetic studies were not possible with this review the similarity of presentation amongst the triplets does indeed suggest a common aetiology, probably genetic. The challenges of diagnosis in a developing country are highlighted in this case, where despite the suspicion of the diagnosis; confirmation is delayed because of logistic reasons. This raises the question if L-thyroxine may be started in suspected cases where a diagnosis cannot be readily established pending a final confirmation. What are the risks and benefits of such a therapeutic approach? This would require further work to clarify.

\section{CONCLUSION}

Congenital hypothyroidism can occur in multiple order pregnancies. There should be a high index of suspicion where facilities for neonatal screening are not available.

Indirect Method for the Assessment of Iodine Deficiency in Estonia. Hormone Research in Paediatrics, 52(6): 284-286.

Olivieri, A., Medda, E., De Angelis, S., Valensise, H., De Felice, M., Fazzini, C., Cascino, I., Cordeddu, V., Sorcini, M. and Stazi, M. A. (2007). High Risk of Congenital Hypothyroidism in Multiple Pregnancies. The Journal of Clinical Endocrinology \& Metabolism, 92(8): 31413147.

Park, S. M. and Chatterjee, V. K. K. (2005). Genetics of congenital hypothyroidism. Journal of Medical Genetics, 42(5): 379-389. 